\title{
Comparing Relativistic Theories Against Observed Perihelion Shifts of Icarus and Mercury
}

\author{
Steven D. Deines \\ Donatech Corporation, Inc., Fairfield, USA \\ Email address: \\ sddeines@hotmail.com \\ To cite this article: \\ Steven D. Deines. Comparing Relativistic Theories Against Observed Perihelion Shifts of Icarus and Mercury. International Journal of \\ Applied Mathematics and Theoretical Physics. Vol. 3, No. 3, 2017, pp. 61-73. doi: 10.11648/j.ijamtp.20170303.14
}

Received: October 11, 2016; Accepted: January 3, 2017; Published: May 28, 2017

\begin{abstract}
This paper compares the post-Newtonian approximation (PNA) to general relativity (GR) for the relativistic perihelion shift calculations. Nelson's PNA predicts 5/6 of GR's perihelion shift. Using the original Universal Time (UT), Shapiro's accurate, highly elliptical orbit for Icarus corroborates PNA while GR exceeds the error boundary. The Icarus result was $\lambda=0.75 \pm 0.08$ where $\lambda=1$ for GR and $\lambda=0$ for Newtonian theory. Studies of Mercury's perihelion shift used timescales equivalent to lunar Ephemeris Time (ET) with the present Système International (SI) second, the basic time unit for all atomic timescales like International Atomic Time (TAI). Atomic timescales run faster than UT, because the SI second is $2.468 \mathrm{E}-8 \mathrm{~s}$ shorter than the original UT second. This is confirmed by the two observational reports using the original calibration data of 1955-1958, by the Improved Lunar Ephemeris used in the original calibration, by the linear divergence of TAI versus UT during 1958-1998, and by the 2.1 ms mean excess between a UT day and TAI day during 1958-1998. Time dilation was not included in the lunar theory, which is confirmed by timekeeping authorities. So, the undilated lunar ET second is shorter than Earth's proper UT second. An ET timescale creates an additional, artificial perihelion shift for Mercury of 6.433"/cy. Other renowned relativists used a 1973 update for Earth's general precession that now excludes the GR prediction while including the PNA prediction if the artificial Mercury shift is included in the calculations. Apparently, Nelson's PNA is more accurate than GR.
\end{abstract}

Keywords: Timescales, Perihelion Shift, Post-Newtonian Approximation, Relativity

\section{Introduction}

Einstein derived his special theory of relativity by assuming that the laws of physics are the same in every inertial reference frame and the speed of light in every direction is constant [1]. This theory maintains the same equations for electrodynamics with the Lorentz transformations for all inertial reference frames. Maxwell's equations predict that the speed of light in vacuum is a universal constant, but the Galilean transformation would predict different speeds of light between an absolutely stationary reference frame and a uniformly moving (i.e. inertial) reference frame. Einstein took a crucial step to construct a relativistic theory of gravitation when he introduced the Principle of Equivalence of Gravitation and Inertia $[2,3,4]$. He used it to calculate the red shift of light in a gravitational field. He became dissatisfied with his progress and began collaboration with Marcel Grossman around 1913.
Einstein incorporated the Principle of Equivalence with the metric tensor formalism by requiring the physical equations be invariant under a general coordinate transformation, not just by a Lorentz transformation [5]. During the next two years, Einstein presented to the Prussian Academy of Science various papers that gave the metric tensor and calculated the solar deflection of light and the precession of Mercury's perihelion [5, 6] and later summarized his work in 1916 [7].

Einstein developed three tests of his general relativity (GR): $\{1\}$ perihelion shift of a planet, $\{2\}$ the solar deflection of light and $\{3\}$ the gravitational red shift of light. There can be various relativity theories based on various metrics. The Parameterized Post-Newtonian (PPN) theories rely on ten parameters that were developed by Will and Nordtvedt $[8, \mathrm{p}$. 98-99] and are denoted as $\gamma, \beta, \xi, \alpha_{1}, \alpha_{2}, \alpha_{3}, \alpha_{4}, \zeta_{1}, \zeta_{2}, \zeta_{3}$, and $\zeta_{4}$. If one applies the full conservative laws to any PPN theory (i.e. energy, momentum, angular momentum and center-of-mass motion), then every $\alpha$ and $\zeta$ term must be zero [8, p. 113]. Thus, only three parameters vary between 
PPN theories if conservation laws are applied to PPN gravitational metrics. For example, Einstein's GR theory has $\gamma=\beta=1$ and $\xi=0$ in the PPN formalism [8, p. 115]. The gravitational red shift experiment is a test of Einstein's Principle of Equivalence, and it does not specifically test GR as all PPN theories are based on the Equivalence Principle [8, p. 166].

However, one can derive the red shift formula for the Pound-Rebka experiment without relativity with $\Delta \mathrm{v} \approx \mathrm{gh} / \mathrm{c}$ where $\mathrm{g}$ is Earth's local gravitational acceleration of 9.8 $\mathrm{m} / \mathrm{s} 2, \mathrm{~h}$ is the height between the photon emission and absorption (i.e. the four-story physics building in Harvard), and $c$ the speed of light in a nongravitated vacuum [9]. It has been shown that all freely-falling reference frames are noninertial due to tidal forces existing in any finite domain of such frames [10]. This means the laboratory was accelerated, and the actual speed of light is altered slightly with $\Delta \mathrm{v} \approx \Delta \mathrm{c}$ due to the local gravity. With $\lambda$ as the wavelength, $v$ as the frequency, $\mathrm{h}$ as Plank's constant, then $\Delta \mathrm{c} / \mathrm{c} \approx \Delta(\lambda v) / \lambda v \approx \Delta v / v$ $\approx \mathrm{h} \Delta \mathrm{v} / \mathrm{h} v \approx \Delta \mathrm{E} / \mathrm{E} \approx \mathrm{gh} / \mathrm{c}^{2}$. This is the red shift that was observed in the Pound-Rebka test.

The solar deflection of light from a distant star has been observed to be 1.75 ", with high precision using pulsars, which verifies the GR prediction [11]. Classical physics, when properly derived, can obtain the same prediction for the solar deflection as GR [12]. This leaves the perihelion shift as the last "classical" test of relativity. The precession of the orbital perihelion using a Schwarzschild solution [5, p. 197] is

$$
\Delta \varphi=6 \pi \frac{\mathrm{M}_{\odot} \mathrm{G}}{\mathrm{a}\left(1-\mathrm{e}^{2}\right)}
$$

where a and e are the semimajor axis and eccentricity of the orbit, respectively, G is Newton's gravitational constant, and $\mathrm{M}_{\odot}$ is the mass of the Sun as the planetary masses are insignificant contributors. I. I. Shapiro wanted to test (1) using a highly elliptical orbit to verify the eccentricity dependence within the prediction.

\section{Relativistic Determination of the Orbital Fits of Icarus}

Shapiro et al [13] chose Icarus for its large orbital eccentricity and period with its close approaches to Earth to observe how well its orbit compared to the predicted relativistic effects on perihelion advance. All calculations were performed in a coordinate system whose origin was at the solar barycenter with the axes set by the mean equinox and equator of 1950.0. The day was chosen as the unit of coordinate time $(\mathrm{CT})$ with $\mathrm{CT}=32.15 \mathrm{sec}+\mathrm{A} 1$, where A1 was the atomic time kept at the U. S. Naval Observatory (USNO). The second was $1 / 86400$ of a CT day. The model for Earth's rotational motion included the accepted international nutation and precession matrices for that period, the rotation model included the polar motion offsets for the rotation axis, and the values of Universal Time (UT) were related to A1 through the USNO time service bulletins.
Shapiro converted all observations into UT, since many photographic observations from various observatories were time tagged in Greenwich Mean Time (GMT) - a earlier designation for UT. A dimensionless parameter $\lambda$ was used to determine how well the orbital motion conformed to the predicted effect with the perihelion (unity for GR and zero in Newtonian theory). The researchers received 342 photographs taken by 17 observatories, and the photographic plate accuracy typically achieved $0.1 \mathrm{sec}$ of arc or better.

In the first solution, only the orbital elements of Icarus and $\lambda$ factor were estimated with all other parameters and initial conditions held fixed. The $\lambda$ factor, estimated at $0.75 \pm 0.08$, was allowed to only influence Icarus' orbit while the Earth's orbit for the observer was based on the correctness of GR $(\lambda$ $=1)$. The formal standard error was obtained by assuming each declination and right ascension observation had a standard error of 1 arc sec, although most observers believed the plates were not worse than a few tenths of an arc second. As Shapiro et al wrote,"... either GR was incorrect or something in the theoretical model or observations differed from their presumptions".

So, the search went on to look for the source of the deviation in $\lambda$ from being the ideal 1 . Solutions were obtained with a variety of different subsets of data included in the analysis. When essentially all of the 1968 data were omitted, the result for $\lambda$ changed. Solutions 2 and 3 as shown in Table 1 are typical of those results, which are not near 1.

The estimated parameter set was widened to include the initial conditions of Earth's orbit. Solution 4 was $\lambda=1.10 \pm$ 0.20 . The error was increased mainly by the high correlation (-0.8) between the estimate for $\lambda$ and that for the Earth's semimajor axis, $\mathrm{a}_{\oplus}$. Note that the correlation of increasing $\lambda$ means a decrease in $a_{\oplus}$. A smaller orbit means a smaller period, which, if divided up in the same number of time units, results in shorter seconds. (This possibility agrees with the fact that the atomic second was calibrated to coordinate time with an undilated second, rather than the UT proper timescale and its average UT second.) Shapiro et al estimated a partial set of Earth's orbital elements including its semimajor axis with Icarus' orbital parameters. Solutions 5 and 6 are given in Table 1. However, Earth's orbital parameters are far less well determined through the Icarus data than with the combined radar and optical observations provided by other sources to Shapiro et al. So, other error sources were examined for the low value in $\lambda$. One hypothesis was Icarus could be a comet fragment that had outgassing or dust emissions that might perturb its orbit. As no visible evidence revealed a comet's tail and a nongravitational model would be hard to test, this possibility was not pursued.

The plausible source was the FK4 star catalogue may be severely distorted with respect to a truly inertial system. To test this assumption, three additional parameters were included: equator, equinox and declination biases. Solution 7 includes the equator and equinox parameters added to the original set of seven, which the Icarus data were expressed. Solution 8 included all three reference system parameters 
with the original set of seven. Additional variations in the data set did not affect the results significantly from the results of Solutions 7 and 8 . As a further check on $\lambda$ results, radar and optical observations of the Sun and inner planets were added to the Icarus data and appropriate initial conditions and parameters were added to the estimated parameters. In particular, the mass of Mercury was estimated and separate constants of $\lambda$ were introduced for Icarus', Mercury's, and Venus' orbital motions. Solutions 9 and 10 for $\lambda$ for Icarus are in Table 1 with the three Icarus reference system parameters excluded or included, respectively. Shapiro et al concluded that the anomalously low value for $\lambda$ was due virtually to distortions of the FK4 reference system to get the positions of Icarus.

Table 1. Solutions for $\lambda$ Parameter Using Different Estimation Criteria.

\begin{tabular}{lll}
\hline Solution & $\lambda$ parameter & Estimated Parameters or Solution sets \\
\hline 1 & $0.75 \pm 0.08$ & Icarus' 7 orbital parameters (all data) \\
2 & $0.79 \pm 0.08$ & Icarus' 7 orbital parameters (subset data) \\
3 & $0.73 \pm 0.08$ & Icarus' 7 orbital parameters (subset data) \\
4 & $1.10 \pm 0.20$ & Icarus' 7 orbital parameters Earth's 7 orbital parameters \\
5 & $0.89 \pm 0.19$ & Icarus' 7 orbital parameters Some of Earth's orbital parameters including Earth's semimajor axis \\
6 & $01.23 \pm 0.20$ & Icarus' 7 orbital parameters Some of Earth's orbital parameters including Earth's semimajor axis \\
7 & $0.85 \pm 0.08$ & Icarus' 7 orbital parameters Equator and equinox of reference frame \\
8 & $0.95 \pm 0.08$ & Icarus' 7 orbital parameters Equator, equinox and declination biases \\
9 & $0.80 \pm 0.08$ & Icarus' 7 orbital parameters Add Sun, Mercury and Venus data Exclude reference frame biases \\
10 & $0.96 \pm 0.08$ & Icarus' 7 orbital parameters Add Sun, Mercury and Venus data Include reference frame biases \\
\hline
\end{tabular}

Since that report, FK5 and other star catalogues have been developed. One major concern was to correct the FK4 catalog for any possible distortions in the stellar locations. However, the transformation between FK4 and FK5 star catalogues is a simple rotation matrix that is an equinox correction of +0.035 " in right ascension at B1950 for all stars in the FK4 system to the FK5 system [14, p. 167-169, 505]. The FK5 also corrected the FK4 catalog for proper motions of $0.085 \% / c y$ and precession by $1.10 \% / c y$. The reference frames for each catalogue have orthogonal axes with no distortions found in the FK4 catalogue in any major sectors. Thus, the last four solutions are discounted as the severe FK4 distortion in 3 coordinates does not exist to explain the lower fit of $\lambda$. Shapiro et al had an initial result of $\lambda=0.75 \pm 0.08$, because UT was the proper timescale used in that study. Shapiro el at did not use the A1 timescale (i.e. identified by Shapiro as coordinate time C. T. with a constant offset) in the analysis.

Shapiro and his team are expert relativists and observational astronomers. They crosschecked their results of Icarus to be consistent. Solutions 4, 5, and 6 in Table 1 involve the change in Earth's semimajor axis, which is heavily correlated $(-0.8$ per [13]) to the parameter $\lambda$, meaning if one decreases Earth's orbital period with a shorter semimajor axis in these fits by dividing the orbital year with the same number of second time units, one obtains a closer fit of the Icarus observations to GR's prediction of the perihelion shift using shorter seconds. It will be shown later in this paper that using shorter second time units (e.g. C. T. or equivalently the lunar Ephemeris Time or atomic seconds) than the required proper second time unit (i.e. the original UT second) will obtain an artificial increase in the predicted perihelion advance for Mercury.

\section{Brief History of Universal and Ephemeris Timescales}

The timescale standard used by all civilizations prior to
1900 was apparent solar time that was refined into mean solar time. The Earth's rotation made the length of day vary by the observer's latitude due to the $66.5^{\circ}$ tilt of the Earth's rotational axis with respect to the ecliptic plane and due to the elliptical orbit of Earth revolving around the Sun, which requires the equation of time. Sundials determine apparent solar time, and the variation of the day is corrected for the local latitude and equation of time with an analemma. John Flamsteed, the first Astronomer Royal of the Greenwich observatory, had determined tables of the equation of time around 1667, but delayed publication until 1672. In July 1676, he resided at the Greenwich observatory, and Greenwich Mean Time (GMT) was begun. Flamsteed later determined the Earth's rotation with his tables was more accurate than his best pendulum clocks at Greenwich. So, Earth' rotation was the time standard for over 2 centuries to maintain GMT by taking meridian transits of the stars to obtain the sidereal day and adjusting that with the equation of time to obtain the mean solar day. Pendulum clocks were calibrated and updated regularly to maintain the mean solar day, as Earth's rotation was the standard time regulator. The second was exactly $1 / 86400$ of a mean solar day as the basic time unit. It was assumed that Earth's rotation rate was always uniform.

The term Universal Time (UT) was officially documented at the International Meridian Conference of 1884 [15] through a US congressional act, which called for a "standard of time-reckoning throughout the globe". Resolution 5 stated "That this universal day is to be a mean solar day", and Resolution 4 states that the initial meridian for measuring longitude would be Greenwich. Thus, mean solar time at Greenwich and Universal Time would be synonymous with GMT relative to midnight.

Simon Newcomb, the chief astronomer of the USNO, suspected that Earth's rotational rate was variable, especially when Chandler documented that Earth's spin axis wobbled [16]. Eventually, Earth's rotation was determined to be variable against a few very precise pendulum clocks, and 
later, quartz oscillators. Using over 60000 observations of the solar longitude from 1750 through 1892, Newcomb published the Tables of the Sun [17]. After obtaining the mean solar longitudes by adjusting for the equation of time and the Chandler wobble and fitting a parabola through the data, Newcomb gave the following expression [18] for the geometric mean longitude of the Sun (referred to the mean equinox of date):

$$
L=279^{\circ} 41^{\prime} 48.04^{\prime \prime}+129602768.13^{\prime \prime} T+1.089^{\prime \prime} T^{2} .
$$

$\mathrm{T}$ in the formula is measured in Julian centuries of 36525 ephemeris days since 1900. The length of the UT second is calculated as a fraction of the tropical year beginning at 1900 January $0,12 \mathrm{~h} \mathrm{ET}$, together with the fact that this particular year contained:

$$
\frac{360 \times 60 \times 60}{129602768.13} \times 36525 \times 86400=31556925.9747 \text { seconds. }
$$

Divide 86400 UT seconds per solar day into this number of seconds to get 365.2421987 solar days in the year 1900 . The solar day is based on Earth's rotation with respect to the mean Sun, which varies around the average of 361.01456+ degrees. Sidereal days are in actual 360 degree rotations from fixed star to fixed star. There is exactly one more sidereal day per year than there are solar days, which results in the sidereal day of 1900.0 having $86164.09055+$ s. It is noted that the true of date rotation rate of $7.292115855 \mathrm{E}-5 \mathrm{rad} / \mathrm{sec}$ is derived from (3). For the rotational rate, divide $2 \pi$ radians by the number of seconds in a sidereal day from 1900.0. The important point is time in (2) is UT, because the times were equivalent to GMT, there was no other worldwide time standard, and the observations were compiled over 142 years to fit the frequency term in (2) to that level of precision. It should be noted that (2) includes the tidal friction effect that slowly decelerates Earth's rotational rate, since it was a polynomial fit of observations after correcting for Earth's elliptical orbit through the equation of time. When the stellar meridian transits for UT are adjusted for polar motion (i.e Chandler wobble), the resulting timescale UT1 is obtained. (Hereafter, UT designates the technical timescale UT1.)

In 1948, Clemence published a specific proposal [19] to use (2) as the fundamental standard of UT that would be smoother than direct observations via stellar transits between adjacent days. The International Astronomical Union (IAU) approved (2) as the definition for Ephemeris Time (ET) with its epoch of 1900.0 at the 1958 General Assembly in Moscow. This solar ET is synonymous with UT. Equation (2) is Newcomb's parabola between the solar mean longitudes and UT time tags. A few minor problems with the solar ET are related to technical issues in the determination of a tropical year and a sidereal year, which depend upon the adopted system of astronomical constants, particularly the constant of precession and the constant of aberration [20, p. 82]. The far larger practical issues made it difficult to observe ET by the motion of the Sun and impractical to get a timely precise determination of ET, which may take years after the observations, to set a precise ET time tag. Despite the
Moon's highly complicated, perturbed orbit, the Moon has 13.37 orbits per year and is easily observed against the stellar background for direct determinations of a lunar ET.

Because the behavior of the analogous residuals in the mean longitudes of Mercury, Venus, and the Moon were all correlated with those of the Sun, Spencer Jones and other astronomers and geophysicists became convinced that the error was not in the theories but was connected to UT, as Newcomb and other before him has suspected [21]. Through the efforts of de Sitter and Spencer Jones, the time corrections for the mean longitudes were documented [22, 23 ] with the tidal friction effect of $22.44 " / \mathrm{cy}^{2}$ (as determined from their work by Clemence) was added to the lunar orbit [19]. The Earth's rotational deceleration has been determined independent of timescales, and the first-order calculations of the lunar tidal friction effect is very close to this lunar orbital deceleration term [24].

Brown's lunar theory, which was derived strictly from Newtonian principles with all the modifications listed by Clemence [19], was soon adopted as a second form of ET. To keep this section brief, refer to Deines and Williams [25, Sections $1 \& 2$ ] for details of all the modifications to Brown's lunar theory, which eventually became the Improved Lunar Ephemeris (ILE) [26] used to calibrate the cesium clocks. It fell to Markowitz and Hall of the USNO to obtain, from lunar observations, the values of ET needed to calibrate the cesium atomic clocks that were maintained by Essen and Parry at the National Physical Laboratory (NPL) in England. The calibration effort lasted between 1955.0 through 1958.25. In 1967, the IAU approved the result as the definition of the Système International (SI) second, which is the basic time unit in International Atomic Time (TAI) [27]. Markowitz et al wrote, "A determination of the frequency of cesium in terms of the second of Ephemeris Time (E. T.) was made jointly by the National Physical Laboratory, Teddington, and the U. S. Naval Observatory, Washington. The frequency is $9,192,631,770 \pm 20$ cycles $\mathrm{s}^{-1}$ of $\mathrm{E}$. T. The second of E. T. is identical with the prototype unit of time defined by the International Bureau of Weights and Measures in 1956" [27]. So, the current SI second matches the lunar ET second and provides continuity between the lunar ET and TAI timescales [20]. It was later shown that the lunar ET second and the SI second differed by less than $1 \mathrm{E}-10$ second after 30 years of the calibration [28]. So, for all practical purposes, the author considers the lunar ET second identical to the SI second.

Because Brown's lunar theory was derived strictly from only Newtonian laws including the Newtonian gravitational law, time dilation was never included in the Improved Lunar Ephemeris (ILE). ]. "There was no consideration of relativistic effects in the first applications of the new atomic standards in establishing an atomic time scale." [29]. Deines and Williams computed the time dilation effect that is missing in the ILE and computed the dilated second (alias the original UT or Earth's proper second) is $2.4676 \mathrm{E}-8$ seconds longer than the undilated second (alias the lunar ET or Earth's coordinate second) [25]. The ILE itself corroborates 
this fact [26]. The proof is documented in [25] and repeated here for the convenience of the reader. Two expressions for the Moon's mean longitude are $\mathrm{L}_{\mathrm{M}}$ and ${ }^{*} \mathrm{~L}$, which are based on time in Julian centuries of UT and ET, respectively. It is clearly stated in the ILE that the determination of ephemeris time would be based on $* \mathrm{~L}$, not $\mathrm{L}_{\mathrm{M}}$, in almost all calculations. Considering two lunar positions separated by the sidereal period of the lunar orbit, then $\mathrm{L}_{\mathrm{M}}\left(\mathrm{UT}_{2}\right)$ $\mathrm{L}_{\mathrm{M}}\left(\mathrm{UT}_{1}\right)=2 \pi=* \mathrm{~L}\left(\mathrm{ET}_{2}\right)-* \mathrm{~L}\left(\mathrm{ET}_{1}\right)$. Only considering the linear terms expressed in $\mathrm{L}_{\mathrm{M}}$ and ${ }^{*} \mathrm{~L}$, one obtains approximately the mean motion equation:

$$
\mathrm{n}_{\mathrm{M}}\left(\mathrm{UT}_{2}-\mathrm{UT}_{1}\right)={ }^{*} \mathrm{n}\left(\mathrm{ET}_{2}-\mathrm{ET}_{1}\right)
$$

As $\mathrm{n}_{\mathrm{M}}>{ }^{*} \mathrm{n}$, the time interval $\mathrm{ET}_{2}-\mathrm{ET}_{1}$ must be numerically larger than $\mathrm{UT}_{2}-\mathrm{UT}_{1}$. Since both intervals represent an identical span of time between these two events, the unit of ET must be smaller than the unit of UT to make the time count $\mathrm{ET}_{2}-\mathrm{ET}_{1}$ larger. For arbitrary time intervals, $(\Delta \mathrm{ET}-\Delta \mathrm{UT}) / \Delta \mathrm{ET}=\left(\mathrm{n}_{\mathrm{M}}-{ }^{*} \mathrm{n}\right) / \mathrm{n}_{\mathrm{M}}=2.292 \mathrm{E}-8$. This is close to the calculated time dilation, since (4) considers only linear terms. The conclusion made in [25] is that the lunar ET has a shorter second than the original mean UT second, and the explanation is that time dilation was not compensated in the ILE. The solar ET was defined by a polynomial fit using direct transit observations tagged with the original UT, but the lunar ET ephemeris theory was derived strictly from Newtonian physics that makes no allowance for time dilation.

The very team that precisely calibrated the lunar ET second for the SI (or atomic) second later reported from the same data of lunar observations, cesium frequencies and UT observations that the UT second was longer than the lunar ET second $[30,31]$. The calibration for the cesium clock to the ILE started January 1955 and lasted 3 1/4 years. (For brevity, only [30] will be discussed.) Figure 1 illustrates that the average UT second is longer than the ET second as compared to the standard cesium frequency, which is defined as 9192631770 cycles [27]. (UT2 in Figure 1 is the adjustment made to UT1 for the seasonal changes in the length-of-day interval compared to the mean UT day, but this periodic compensation averages out over a year.) Note that UT seconds are all larger than that standard cycle number for SI.

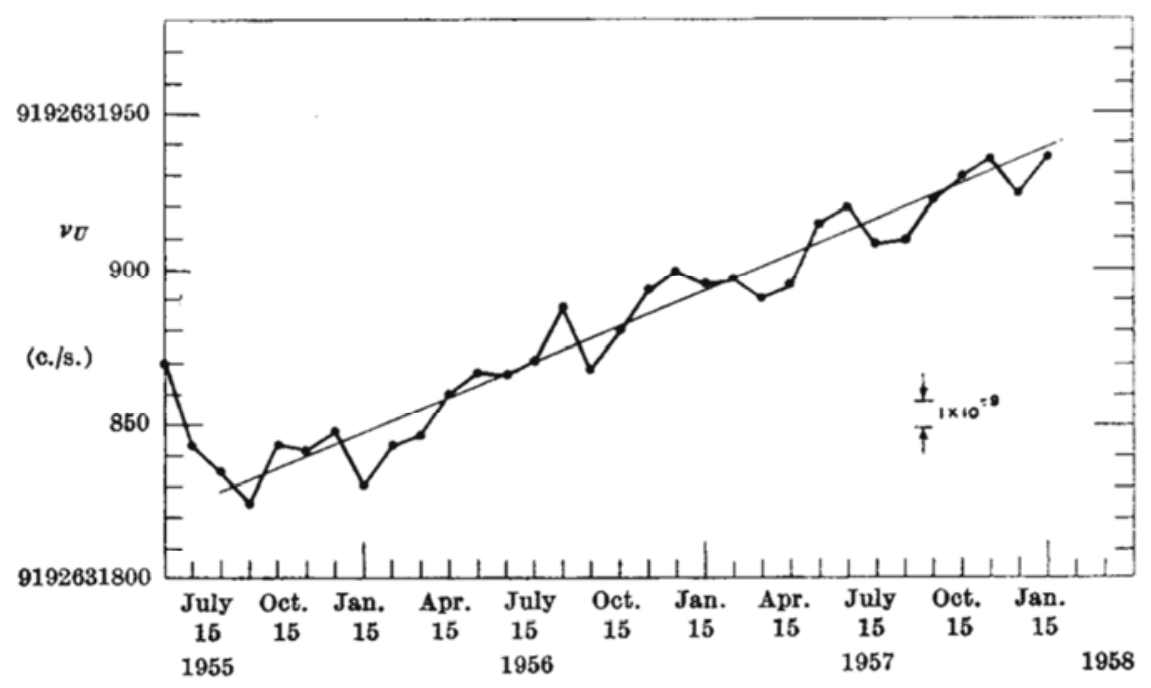

Fig. 1. Frequency of the caesium resonance in terms of UT2.

One can extrapolate some interesting relationships. The UT second is longer than the lunar ET second, so it would take fewer UT seconds than ET seconds to span a given time interval, and the total UT count decreases proportionally to the total ET seconds as the time span increases. The cycles per UT2 second will increase linearly as shown in Figure 1. Between 1955.0 and 1958.25, the elapsed cycles increase for each UT2 second that is accumulated. If 1958.25 (the end of the calibration operation) represents the final UT2 second count, then $(1+2.468 \mathrm{E}-8)$ x $9192631770=9192631997$, which is about $47 \mathrm{c} / \mathrm{s}$ off of the extrapolated 1958.25 intercept in Figure 1.

The size difference between the original UT (alias GMT or solar ET) second and the SI (alias lunar ET or atomic) second can be found in at least 9 different timing anomalies. Again, for brevity, the simplest example is found in the divergence between TAI and UT during the interval of January 1958 through December 1998. TAI was 32 seconds ahead of UT, so there were inserted 32 seconds in the atomic timescale called Coordinated Universal Time (UTC) so that UTC remained within 0.9 seconds of UT. The TAI epoch was set initially to be identical with UT on 0 Jan 1958, but it became readily apparent that TAI was steadily diverging ahead of UT. With 32 seconds over 41 years, the divergence rate is $2.473 \mathrm{E}$ $8 \mathrm{~s} / \mathrm{s}$, which is within $0.2 \%$ of the calculated size difference of $2.4676 \mathrm{E}-8 \mathrm{~s} / \mathrm{s}$ due to time dilation [25]. Also, the size difference between the UT and SI seconds also explains the excess LOD. The mean excess LOD has been $2.1 \mathrm{~ms}$ between 1958 and 1998. Multiply the number of seconds per day by the predicted size difference to get $86400 \mathrm{x}$ ($2.4676 \mathrm{E}-8 \mathrm{~s} / \mathrm{s})=2.132 \mathrm{~ms}$. It has been assumed incorrectly that tidal friction was the cause [20], but Deines and Williams confirmed that tidal friction was accurately compensated within the ILE after showing first-order 
calculations of the conservation of angular momentum transfer to the Moon are nearly the empirical adjustment of the lunar orbital deceleration [24]. So, the cause must lie elsewhere. The divergence of TAI compared to UT between 1958 and 1998 is based on the daily meridian transits of the stars to complete a mean sidereal day, and the meridian transit observations using Photographic Zenith Tubes (PZT) at Washington, DC, and Richmond, FL, are completely described in the processing by Markowitz [32]. The important point is the operational epoch for UT was advanced daily except for very infrequent cloudy conditions at both locations. This allowed the daily observations in the UT excess length-of-day (LOD) beyond an atomic day over decades, which periodic variations were found in daily, weekly, semimonthly, monthly, bimonthly, seasonal, semiyearly, yearly, and decadal intervals. Deines and Williams determined that tidal friction would be 12 orders too small to be detected in daily data (2E-15 s in Earth's rotation after 1 day compared to the mean $2.1 \mathrm{~ms}$ LOD difference between UT and TAI). The International Earth Rotation Service changed the processing of UT by adopting Very Long Baseline Interferometry (VLBI) data from radio telescopes and new formulas and processing in 1999 to replace the PZT meridian transit operations. Unfortunately, no overlap of operations was allowed due to budget cuts to verify that the new VLBI processing ever replicated the accuracy of the PZT meridian transit operation for determining UT.

When one uses TAI as the timescale instead of the original UT timescale, there will be $77.87 \mathrm{~s}$ difference between the timescales after a Julian century $(36525$ UT days $x$ $86400 /$ day $\mathrm{x}-2.4676 \mathrm{E}-8 \mathrm{~s} / \mathrm{s})$. This will cause an artificial perihelion shift for Mercury to advance $6.433 \% /$ cy more, which will be explained in Section 5 .

\section{Post-Newtonian Approximation Compared to General Relativity}

Initially, the observed Mercury perihelion shift of 42.56\%/cy reported by Clemence [33, 34] in 1943 and 1947 nearly fits the 43.03 "/cy predicted by GR. Clemence wrote [34], " $T$ is the time required for one revolution of the planet... and T [is] measured in seconds." Unfortunately, he was completely silent in both papers whether he used UT or Ephemeris Time (ET) for the timescale. The most likely option was Clemence used ET for his analysis. In 1948, Clemence presented the formulation and theory that defined ephemeris time as a timescale (although he initially called it Newtonian Time) by using Newcomb's formula for the mean motion of the apparent Sun that was calibrated from decades of observations [19]. The empirical formula predicted the Sun's apparent position with the independent parameter as time, which the formula was actually Earth's ephemeris revolving around the Sun in the solar system reference frame with the origin at the solar barycenter (i.e. center of mass). The formula could be used in reverse to assign a time given the observed position of the Sun, hence the name Ephemeris Time (ET).

The PNA that is appropriate for an observer on Earth's surface [35] is compared with GR for evaluating a key relativistic test-perihelia shift. Weinberg [2, p. 230-232] gave a proof that his Einstein term (9.5.17) produces the identical result as GR for the precession of perihelia, which he designated as $\phi_{0}$. Equating his (9.5.16) to the final result of (9.5.17), the precession per revolution is:

$$
\Delta \varphi_{0}=\int_{0}^{2 \pi} \frac{d \varphi_{0}}{d t} \frac{d y}{d x} d \varphi=6 \pi \frac{M_{\odot} G}{L}
$$

where $\mathrm{L}$ is the semilatus rectum of the orbit, $\mathrm{G}$ is the gravitational constant, and $\mathrm{M}_{\odot}$ is the mass of the Sun [2, p.232]. Weinberg wrote, "Since $\varphi_{0}$ changes slowly, the change in $\varphi_{0}$ in one revolution can be determined by integrating $d \varphi_{0} / d t$ over one period, keeping $\phi_{0}$ fixed in the integrand, and using for $d \varphi / d t$ the Keplerian formulas." [2, p.232]. The integration of $d \phi$ over one revolution is relative to a fixed inertial frame, but $d \varphi_{0} / d t$ is positive in observations of Mercury's perihelion. Integration over one orbit may be tiny, but integration spanning a century of 415 orbits of Mercury is not insignificant. Weinberg also wrote, "If $d \varphi_{0} / d t$ is positive, then the precession is in the same sense as the direction of the planet's motion" [2, p.231]. This confirms $d \varphi_{0} / d t$ advances in the same direction as $d \phi / d t$, which Weinberg's proof strictly means $\Delta \varphi \equiv \varphi-\varphi_{0}<$ $6 \pi M_{\odot} G / L$. This means the PNA is strictly less than the GR prediction. The perihelia value from the post Newtonian approximation will be evaluated next.

\section{Relativistic Time Dilation Using a Post-Newtonian Metric}

Nelson derived the general relativity (GR) space-time metric and equation of motion with respect to an accelerated, rotating frame of reference in the presence of a gravitational field in a post-Newtonian approximation (PNA). He applied the results to obtain a test particle's motion with many mass points as generalized by the Einstein-Infield-Hoffmann (EIH) equation of motion for a noninertial observer. The complete metric combined gravitational and inertial effects as a solution to the field equations. The gravitational terms are not simply added, due to the gravitational-inertial coupling. The metric was fully derived without a simple rotation of axes used in other post-Newtonian approximations. This metric fully applies to an observer on Earth's surface. There is no restriction, rotational or translational, on the motion of the observer's coordinates [35]. Nelson derived the relativistic equation of motion, but not time dilation for a rotating, accelerated observer.

Two timescales found in Einstein's theory of special relativity are proper time, $\tau$, that is, the time kept by an observer, and coordinate time, $t$, the uniform independent variable of the equations of celestial mechanics. According to Einstein, an inertial frame is a coordinate system where Newton's mechanical laws hold and is equivalent to 
Einstein's definition of a resting frame [36]. In celestial mechanics, the barycenter of the solar system usually represents the origin of the nonaccelerating reference frame to which orbital motions are referred. If Einstein is correct, the Newtonian equations of Mercury's solar orbit are correct for a closed orbit (without external perturbations) in Mercury's inertial frame using Mercury's proper time. An Earth observer's proper time would not match Mercury's proper time, which would cause an apparent shift in Mercury's perihelion due to the difference in time units due to the difference in gravitational potentials, dynamical accelerations and orbital velocities.

Adapt the work of Nelson [35] to establish the proper time of the observer. Nelson developed a post-Newtonian metric with signature $(+,+,+,-)$, which sets $g_{00}<0$. Following Ohanian \& Ruffini [37, p. 322], take $d s^{2}=-c^{2} d \tau^{2}$, where $s$ is the space-time arc length and $c$ is the speed of light in a vacuum. Nelson established the components of the metric tensor for an observer undergoing translational and rotational accelerations and subject to both scalar and vector gravitational potentials. These conditions are typical for an observer on the surface of the Earth orbiting around the barycenter of the solar system.

Nelson used an iterative solution of Einstein's field equations to obtain directly the generalized metric containing gravitational, inertial and mixed gravitational-inertial terms. In this generalized derivation, space-time was assumed to be locally curved and gravitational effects of local matter were explicitly described in terms of the Newtonian potential and standard post-Newtonian scalar and vector potentials. The resulting post-Newtonian space-time metric $g_{\alpha \beta}$ to order 4 is:

$$
\begin{gathered}
g_{i j}=\left(1-2 \phi / c^{2}\right) \delta_{i j}+\mathrm{O}\left(\varepsilon^{4}\right) \text { for a Cartesian frame } \\
g_{0 j}=\left(1-2 \phi / c^{2}\right)(\omega \times r)_{j} / c+\mathrm{U}_{j} / c^{3}+\mathrm{O}\left(\varepsilon^{5}\right) \\
-g_{00}=\left(1+\mathbf{W} \cdot \mathbf{r} / c^{2}\right)^{2}+2 \varphi / c^{2}+2 \varphi^{2} / c^{4}+4 \Psi / c^{4}-\left(1-2 \varphi / c^{2}\right)(\boldsymbol{\omega} \times \mathbf{r})^{2} / c^{2} \\
-2(\boldsymbol{\omega} \times \mathbf{r}) \cdot U / c^{2}+4 \varphi(\mathbf{W} \cdot \mathbf{r}) / c^{4}+(\mathbf{W} \cdot \operatorname{grad} \chi) / c^{4}+\mathrm{O}\left(\varepsilon^{6}\right)
\end{gathered}
$$

where $\mathbf{W}$ is the time-dependent translational, acceleration of the observer's frame relative to an inertial frame,

$\boldsymbol{\omega}$ is the time-dependent angular velocity vector of the observer's spatial frame rotating relative to the inertial frame,

$\phi$ is the classical Newtonian gravitational potential external to the observer, and

$\mathbf{r}$ is the range vector from the accelerated observer's origin to any object.

In the accelerated, rotating frame, the potentials $U, \chi$, and $\Psi$ are further defined in Nelson's paper [35], but these terms do not contribute to second order of $\mathrm{c}$ to develop the time dilation equation.

Let the inertial frame be at the solar barycenter where the gravitational potential $\phi=-\mu / \mathrm{r}$ with the reduced mass $\mu$ by ignoring the other gravitational perturbations due to other bodies in the solar system. The translational centripetal acceleration is $\mathbf{W}=-\mu \mathbf{r} / \mathrm{r}^{3}$. Retain the first and second order terms with three fourth order terms to get the following metric:

$$
\begin{gathered}
g_{i j}=\left(1-2 \varphi / c^{2}\right) \delta_{i j}+\mathrm{O}\left(\varepsilon^{4}\right) \approx\left(1-2 \varphi / c^{2}\right) \delta_{i j} \quad \text { [Only } g_{i j} \approx \delta_{i j} \text { actually contributes.] } \\
g_{0 j}=\left(1-2 \varphi / c^{2}\right)(\boldsymbol{\omega} \times \mathbf{r})_{j} / c+\mathrm{U}_{j} / c^{3}+\mathrm{O}\left(\varepsilon^{5}\right) \approx(\boldsymbol{\omega} \times \mathbf{r})_{j} / c \\
-g_{00}=\left(1+\mathbf{W} \cdot \mathbf{r} / c^{2}\right)^{2}+2 \varphi / c^{2}+\varphi^{2} / c^{4}-(\boldsymbol{\omega} \times \mathbf{r})^{2} / c^{2}+2 \varphi(\mathbf{W} \cdot \mathbf{r}) / c^{4}+\mathrm{O}\left(\varepsilon^{4}\right) \\
\approx\left(1+\frac{\mathbf{W} \cdot \mathbf{r}+\varphi}{c^{2}}\right)^{2}-\frac{(\boldsymbol{\omega} \times \mathbf{r})^{2}}{c^{2}}
\end{gathered}
$$

Substitute the above metric directly into the metric equation.

$$
d s^{2}=\left(1-2 \varphi / c^{2}\right) \delta_{i j} d x^{i} d x^{j}+\frac{2}{c}(\boldsymbol{\omega} x \mathbf{r})_{j} d x^{j} d x^{0}-\left(\left(1+\frac{\mathbf{W} \cdot \mathbf{r}+\Phi}{c^{2}}\right)^{2}-\frac{(\boldsymbol{\omega} x \mathbf{r})^{2}}{c^{2}}\right) d x^{0} d x^{0}
$$

Substitute $d x^{0}=c d t$ and apply the chain rule.

$$
d s^{2}=-\left\{\left(1+\frac{\mathbf{W} \cdot \mathbf{r}+\varphi}{c^{2}}\right)^{2}-\frac{(\boldsymbol{\omega} x \mathbf{r})^{2}}{c^{2}}-\frac{2}{c^{2}}(\boldsymbol{\omega} x \mathbf{r})_{j} \frac{d x^{j}}{d t}-\left(1-2 \varphi / c^{2}\right) \frac{\delta_{i j}}{c^{2}} \frac{d x^{i}}{d t} \frac{d x^{j}}{d t}\right\} c^{2} d t^{2}
$$

Note again that $\left(\frac{d r}{d t}\right)^{j}=\frac{d x^{j}}{d t}$, and $\delta_{i j} \frac{d x^{i}}{d t} \frac{d x^{j}}{d t}=\left(\frac{d r}{d t}\right)^{2}$ for $i \neq 0$. 


$$
d s^{2}=-c^{2}\left\{\left(1+\frac{\mathbf{W} \cdot \mathbf{r}+\varphi}{c^{2}}\right)^{2}-\frac{1}{c^{2}}\left(1-2 \varphi / c^{2}\right)\left(-\boldsymbol{\omega} x \mathbf{r}-\frac{d \mathbf{r}}{d t}\right)^{2}\right\} d t^{2}=-c^{2} d \tau^{2}
$$

Divide by $\mathrm{c}^{2}$ and use the relationship for the rate of change of a vector,

$$
\mathbf{v}_{\text {inertial }}=\left(\frac{d \mathbf{r}}{d t}\right)_{\text {inertial }}=\left(\frac{d \mathbf{r}}{d t}\right)_{b o d y}+\boldsymbol{\omega} x \mathbf{r}=\mathbf{v}_{b o d y}+\boldsymbol{\omega} x \mathbf{r}
$$

Ignore all fourth order terms, which are numerically insignificant to the second order terms in the weak gravitational potential and with low velocities. The above equation is reduced to

$$
d \tau^{2}=\left\{\left(1+\frac{\mathbf{W} \cdot \mathbf{r}+\varphi}{c^{2}}\right)^{2}-\frac{v^{2}}{c^{2}}\right\} d t^{2}
$$

Take the square root approximation to second order and obtain:

$$
\tau=\int_{0}^{T}\left[1+\frac{\varphi_{\text {Gravity }}}{c^{2}}+\frac{\mathbf{W} \cdot \mathbf{r}}{c^{2}}-\frac{v^{2}}{2 c^{2}}\right] d t
$$

Several assumptions are adopted to simplify Equation (6): 1. the origin of the inertial frame is at the center of mass of the Sun by ignoring the planetary masses and any planetary perturbations to the orbits, 2. Mercury is reduced to a point mass located at Mercury's center of mass, 3. the scalar potential is evaluated using the Sun's gravity field only, and 4. a Keplerian ellipse is the model for $\mathbf{W}$. From the last assumption, $\mathbf{W}=\ddot{\mathbf{r}}=-\mu \mathbf{r} / \mathrm{r}^{3}$ and $\mathbf{W} \cdot \mathbf{r}=\varphi=-\mu / \mathbf{r}$, where $\mu$ is the reduced mass between the Sun and Mercury. For the elliptical orbit, integration is best done using the eccentric anomaly E. The pertinent equations after solving Kepler's problem for an elliptical orbit are:

$$
\mathrm{v}^{2}=\frac{\mu}{\mathrm{a}} \frac{1+\mathrm{e} \cos \mathrm{E}}{1-\mathrm{e} \cos \mathrm{E}}, \sqrt{\frac{\mu}{\mathrm{a}^{3}}}\left(\mathrm{t}-\mathrm{t}_{\text {perihelion }}\right)=\mathrm{E}-\mathrm{e} \sin \mathrm{E}, \text { and }
$$

$r=a(1-e \cos E)$ where $a$ is the semimajor axis and $e$ is eccentricity. Substitute $v^{2}, r$ and the derivative of $E$ with respect to $t$. Take the definite integral over one anomalistic period with $\tau_{0}=\mathrm{t}_{\text {perihelion }}$ at 0 radians and $\mathrm{t}=1$ orbital period at $2 \pi$ radians to get:

$$
\left.\tau-\mathrm{t}=-\frac{5}{2} \frac{\sqrt{\mu \mathrm{a}}}{\mathrm{c}^{2}} \mathrm{E}\right]_{0}^{2 \pi}=-5 \pi \frac{\sqrt{\mu \mathrm{a}}}{\mathrm{c}^{2}}
$$

Using the heliocentric gravitational constant for $\mu$ (since Earth's mass is 6 orders smaller than the Sun's) and the mean distance of Earth's orbit (1 Astronomical Unit in meters) [20], the Earth's time dilation is $-0.77875 \mathrm{~s}$ per anomalistic year as published [25]. Adjusting the result by the standard tropical year (365.24219/365.25964), the time dilation for Earth is $-0.77871 \mathrm{~s}$ of lunar ephemeris time per tropical year [25]. Use (7) to calculate Mercury's time dilation for an Earth observer, which causes the observable perihelion shift that Newtonian physics does not include. The semimajor axis for Mercury is 0.387098 A. U., and Mercury's $\tau-\mathrm{t}=$
$-0.48449 \frac{\mathrm{s}}{\text { orbit }}=-6.3744 \mathrm{E}-8$ revolution/orbit as Mercury has a period of 87.96925 days in terms of Earth's tropical year (365.2421987 days) [20].

$$
\begin{gathered}
\tau-\mathrm{t}=-4.0052 \mathrm{E}-7 \frac{\mathrm{rad}}{\text { orbit }} \times \frac{1 \text { Mercury orbit }}{87.96925 \mathrm{~d} \times 86400 \mathrm{~s} / \mathrm{d}} \\
\tau-\mathrm{t}=-5.2696 \mathrm{E}-14 \frac{\mathrm{rad}}{\mathrm{s}} \times \frac{180^{\circ}}{\pi \mathrm{rad}} \times \frac{3600^{\circ}}{1^{\circ}} \times \frac{100 \mathrm{yr}}{\mathrm{cy}} \\
\times \frac{365.2422 \mathrm{~d}}{\mathrm{yr}} \times \frac{86400 \mathrm{~s}}{\text { day }} \\
(\tau-\mathrm{t})_{\text {Mercury }}=-34.30 \mathrm{~N} / \text { cy or }(\mathrm{t}-\tau)_{\text {Mercury }}= \\
34.30 \mathrm{l} / \mathrm{cy}
\end{gathered}
$$

Weinberg gives the general relativistic shift of perihelia (1) as $\Delta \varphi=\frac{6 \pi \mathrm{R}}{\mathrm{a}\left(1-\mathrm{e}^{2}\right)}$ where the gravitational radius $\mathrm{R}=\frac{\mathrm{GM}}{\mathrm{c}^{2}}=\frac{\mu}{c^{2}}$. Kepler's third law provides a parameter change by $\mu=$ $4 \pi^{2} \mathrm{a}^{3} / \mathrm{T}^{2}$. Substitute for $\mu$ to get the resulting equation [38]:

$$
\Delta \varphi=\frac{24 \pi^{3} a^{2}}{T^{2} c^{2}\left(1-e^{2}\right)}
$$

The PNA in Equation (7) is modified by replacing the expression for $\mu$. The undilated time interval will cause the $t$ timescale to run faster than the $\tau$ proper timescale, so:

$$
t-\tau=\frac{10 \pi^{2} a^{2}}{T c^{2}}
$$

Use the relationship of mean anomaly versus orbital period, where $2 \pi$ radians of mean anomaly $=\mathrm{T}$ in seconds for orbital period. The time dilation can be converted to an angle of precession by:

$$
\Delta \varphi=\varphi-\Phi=(\mathrm{t}-\tau) \frac{2 \pi}{\mathrm{T}}=\frac{20 \pi^{3} \mathrm{a}^{2}}{\mathrm{~T}^{2} \mathrm{c}^{2}}
$$

Except for eccentricity, which is near zero for Mercury's orbit, the ratio of the PNA (10) to the GR prediction (9) is 5/6. The PNA for an Earthbound observer will predict a perihelion shift that is $5 / 6$ of the GR prediction, as actually proven by Weinberg.

This is significantly less than the GR prediction of 42.98 "/cy for Mercury \{40]. Clemence reported that the observed excess in Mercury's perihelion was 42.56"/cy in two separate papers in 1943 and 1947, but he was silent on which timescale he used to compile the data $[33,34]$. Clemence used Newcomb's general precession value with an 1850.0 epoch $[33,34]$, but the updated masses of the planets since his publications have changed that term. Misner, Thorne and Wheeler, three renown relativists, updated Clemence's result 
using the 1973 value for the general precession, which produces a result of $(41.4 \pm 0.90)$ "/cy [39, p. 1113]. However, they did not discuss what timescale was used. It is also interesting that they did not remark why the GR prediction is outside their error bounds other than quote Clemence's remark, "The observations....are affected by the precession of equinoxes, and the determination of the precessional motion is one of the most difficult problems of observational astronomy, if not the most difficult" [39, p. 1113].

Morrison and Ward, two excellent observational astronomers, reported that Mercury's perihelion shift was (41.9 \pm 0.5$)$ "/cy by using observations between 1677 and 1973 [40]. They discussed in complete detail that "the observed times of contact are reduced to Universal Time (UT), whereas the argument of time in the dynamical theories of the orbital motions of the Earth and Mercury is nominally Ephemeris Time (ET)". They used the notation ET(Sun) to indicate explicitly ephemeris time in terms of the Sun's mean longitude and the term $\Delta \mathrm{T}(\mathrm{Sun})=\mathrm{ET}(\mathrm{Sun})-\mathrm{UT}$. This $\Delta \mathrm{T}$ (Sun) is expected, because UT has many periodic variations (daily, weekly, semimonthly, monthly, bimonthly, seasonal, yearly, decadal) that deviate from the mean motion of the Sun and average out over the long term. Newcomb had developed the mean motion formula from the Sun's mean longitudes tagged in UT [18] that Clemence recommended as a smoothed timescale, later designated ET(Sun) [19]. As already covered in Section 2, the mean UT timescale and ET(Sun) are the same over the short term (less than 2 centuries). In the tidal friction analysis that the rotational deceleration was $59.358 \mathrm{E}-8 \mathrm{rad} / \mathrm{y}^{2}$ [24], the excess sidereal LOD produces an angular divergence of $1.3359 \mathrm{E}-2 \mathrm{rad}$, and it would take 212 years for tidal friction to overtake that linear divergence [25].

Morrison and Ward also denoted that $\Delta \mathrm{T}$ (Moon) $=$ ET(Moon) - UT. They stated the following correction for $\Delta \mathrm{L}$, derived from Spencer Jones's corrections, were added to the ILE to bring the timescale of the lunar ephemeris, ET(Moon), to agree with the solar ephemeris, ET(Sun).

$$
\Delta \mathrm{L}=-8.72-26.74 \mathrm{~T}-11.22^{\prime \prime} \mathrm{T}^{2}
$$

where T denotes Julian centuries (36525 UT days) since the epoch at noon of 0 January 1900 Greenwich. As explained by Deines and Williams [25], equation (11) is $\Delta \mathrm{L}=* \mathrm{~L}-\mathrm{L}_{\mathrm{M}}$ that was discussed in Section 2 of this paper to generate the ILE. In the early $20^{\text {th }}$ century, the best theories were from S. Newcomb for the Sun and four inner planets [17] and from E. W. Brown for the Moon [41]. In 1926 and again in 1939, Spencer Jones analyzed the residuals between these theories and observations and presented the formulae for the correction of the calculated mean lunar longitudes compared to the solar mean longitudes [25].

Morrison and Ward performed a comparison between $\Delta \mathrm{T}$ (Sun) and $\Delta \mathrm{T}$ (Moon). Their observational equation of condition could not be solved because the constant and secular parts of $\Delta \mathrm{T}$ (Sun) were highly correlated with two parameters that needed to be solved. They avoided this by substituting (11) for the values of $\Delta \mathrm{T}$ (Moon) for $\Delta \mathrm{T}$ (Sun). They assumed that the constant and linear parts of (11) were correct and introduced an unknown variable for $\mathrm{T}^{2}$. They hoped the lunar deceleration term would be more negative than $-22.44 \% / \mathrm{cy}^{2}$ and started with the value $-42.44 " / \mathrm{cy}^{2}$, which Ward had obtained from earlier research. The increase in the sum of squares for the loss of one degree of freedom was significant, so they concluded that the most likely result from all the data for the Moon's tidal acceleration is in the range of ($26 \pm 2) \% / c^{2}$. They also admitted that there are systematic biases in the timing of the observations, but the timing of the transits with the increase resolving power of telescopes would tend to alter the timing of the contacts by changing the observed duration, but not the overall mean time. Those data could not be reconciled with a value of tidal acceleration, and changes in the values of the orbital elements of Mercury and Earth did not alter their conclusion [40]. They used an updated value for the planetary masses [40] and for the general precession determined by Fricke [42] than the values used by Clemence $[33,34]$. In the final result, they used the lunar ephemeris timescale, as it was smoothed compared to the nonuniform UT timescale when reducing the observations.

It is interesting to note that the linear term in (11) can be compared to the sidereal mean motion of the Moon (2.661699489E-6 rad/s) [14]. The term $-26.74 \% /(J u l i a n ~ c y)=$ $4.108 \mathrm{E}-14 \mathrm{rad} / \mathrm{s}$. The ratio between this term and the sidereal mean motion of the Moon is $1.543 \mathrm{E}-8 \mathrm{~s} / \mathrm{s}$, which is close to the time dilation effect between the two seconds that has been calculated [25]. The problem is that Morrison and Ward substituted (11) for $\Delta \mathrm{T}$ (Sun) to solve for the observational equation, which means that the ET(Sun) timescale has been replaced by ET(Moon). Morrison and Ward have inadvertently replaced the original UT timescale (alias ET(Sun)) with the lunar ET timescale, which has a shorter second for a time unit.

The earliest observation of Mercury's perihelion shift came from Urban Le Verrier, director of the Paris Observatory, who announced in 1857 that the Mercury perihelion was off by $38 \% /$ cy after analyzing several decades of observations of Mercury [43]. Le Verrier also predicted in 1846 the location of Neptune based on the perturbations seen in the orbit of Uranus. Although one may argue that the astronomical constants used in the $19^{\text {th }}$ century, including the planetary masses, were not accurate as today's values, Le Verrier's calculations could not be that far in error due to his precision to predict Neptune's location. Newcomb confirmed the excess of 39"/cy in Mercury's observed perihelion shift [18]. Newcomb was then the world's leading astronomer and made many significant contributions to timekeeping, applied mathematics, and planetary astronomy. His results concerning Mercury's perihelion are short of the predictions of GR, which points to a timing issue between timescales. When Morrison and Ward used the updated values for the planetary masses and general precession in Newcomb's theoretical development while using a lunar ET timescale, their results were about 2"/cy less than Clemence's results of 42.56 "cy. With those updated values, Newcomb would have probably obtained an excess of 37\%/cy in Mercury's observed perihelion shift with the original UT. 


\section{Artificial Increase in Mercury Perihelion due to Different Sized Seconds}

Morrison and Ward used ET as a smoothed timescale, but they replaced ET(Sun) for ET9Moon) when they solved for the operational equation of conditions. The timescale that Clemence used to publish his 1947 results for Mercury's perihelion shift was probably in ET. He not only proposed ET(Sun) based on (2) as a smooth timescale for UT, but he also recommended the appropriate, detailed changes in the same publication to Brown's lunar theory, which lead eventually to the ILE being used to calibrate the atomic second [19, 25]. Thus, the actual timescale used by Clemence, by Misner, Thorne and Wheeler, and by Morrison and Ward was the lunar ET timescale.

Aoki [44] and Clemence and Szebehely [45] identified UT as one type of proper time, $\tau$, and associated ET as coordinate time, t. Since the atomic clocks were calibrated to the lunar ET via ILE by 1958, the SI second of TAI is equal for all practicality $(<10 \mathrm{E}-10 \mathrm{~s})$ to the lunar ET second after 30 years [28].

If the observer was using the undilated timescale with the shorter coordinate second (e.g. TAI) to observe Mercury instead of the proper timescale with the original second (i.e. original UT1), which was used in all observations prior to 1955 before atomic clocks, then UT would be behind TAI by $77.871 \mathrm{~s}$ per Julian century (excluding tidal friction that is eventually significant over the long term). The calculated perihelion from the formula would be further advanced than anticipated when using a shorter time interval than the average UT second for proper time. There would be more time units with a shorter second than the original second for a specific time span. Using (8), the addition shift is:

$$
\begin{aligned}
& \Delta(\tau-\mathrm{t})_{\text {Mercury }}=-5.2696 \times 10^{-14} \frac{\mathrm{rad}}{\mathrm{s}} \times \frac{77.871 \mathrm{~s}}{\mathrm{cy}} \\
& \times \frac{87.96925 \times 86400 \mathrm{~s}}{\text { Mercury orbit }} \times \frac{180^{\circ} \times 3600^{\prime \prime}}{\pi \operatorname{rad} \times 1^{\circ}} \\
& \Delta(\tau-\mathrm{t})_{\text {Mercury }}=-6.433^{\prime \prime} / \operatorname{cy} \text { or } \Delta(\mathrm{t}-\tau)_{\text {Mercury }}= \\
& 6.433 " / \mathrm{cy}
\end{aligned}
$$

The point is that the shorter second will produce a shift of $6.433 \% / c y$ in addition to the actual perihelion shift for Mercury. Apply this additional shift to the PNA, and one obtains:

$$
\begin{gathered}
(\mathrm{t}-\tau)_{\text {Mercury }}+\Delta(\mathrm{t}-\tau)_{\text {Mercury }}=34.300 " / \text { cy }+ \\
6.433 " / \text { cy }=40.73 " / \text { cy }
\end{gathered}
$$

This falls within the Misner, Thorne and Wheeler calculation of $(41.4 \pm 0.90) \% /$ cy [39, p. 1113] by modifying Clemence's result with the updated Earth general precession. Clemence's result was $(42.56 \pm 0.94) \% / \mathrm{cy}$, but his error boundary is far too optimistic, because he had computed with less accurate planetary masses and general precession, which have been subsequently replaced by 1973 with newer values.
Clemence was instrumental in the theoretical development of the ILE, so he most likely used a lunar ET for a smooth lunar model, since Brown was working with USNO to improve the lunar model [25]. After subtracting (12), Morrison and Ward's result would be $(35.5 \pm 0.5) \% /$ cy. However, they only expected that an error existed with the tidal friction value, which they computed from a least squares analysis of the quadratic term only. They did not anticipate a linear divergence between ET(Sun) and ET(Moon), although they did describe a consistent timing anomaly in their solution of the operational equation. So, their error boundary is also too small. Still, the modified result of $35.5 \% / \mathrm{cy}$ is only $1.2 \% / \mathrm{cy}$ different than the result of 34.3"/cy in (8). Finally, Shapiro's result for the Icarus perihelion result was $\lambda=(0.75 \pm 0.8)$, which Shapiro definitely used UT as the timescale. He defined $\lambda$ as the ratio $\lambda$ of the numerical result compared to GR where $\lambda=1$ for GR and $\lambda=0$ for Newtonian physics. The PNA is 5/6 of GR, which places it within Shapiro's error bounds. Thus, two out of three studies concerning the perihelion shift in orbits agree with the PNA, which is $5 / 6$ of the GR prediction. The study from Morrison and Ward would probably agree with the other studies and include the PNA if Morrison and Ward realized that the lunar ET second is shorter than the original UT second and if they used the 1973 general precession update instead of their 1967 value.

\section{Conclusion}

Einstein's general relativity (GR) theory predicts that an additional perihelion shift of an orbiting body rotating around the Sun that is not accounted by Newtonian physics will be $\Delta \varphi=6 \pi \mathrm{G} \mathrm{M}_{\odot} /\left(1-\mathrm{e}^{2}\right)$ a, where $\mathrm{G}$ is Newton's gravitational constant, $\mathrm{M}_{\odot}$ is the Sun's mass (as other masses in the solar system are insignificant in comparison), e is the eccentricity of the orbit, and a is the semimajor axis. Shapiro wanted to test the perihelion shift's dependency from eccentricity and chose the asteroid Icarus with its highly eccentric orbit and good visibility to Earth. His team conducted an optical campaign and found that $\lambda=0.75 \pm 0.08$ [11], which $\lambda$ is the ratio of the observed extra perihelion shift compared to GR (i.e. $\lambda=1$ for GR and $\lambda=0$ for Newtonian physics). As Shapiro et al documented, "either GR was incorrect or something in the theoretical model or observations differed from their presumptions" [11]. They concluded that the FK4 star catalog was warped in the sectors that Icarus was observed against, so that the data would appear to be less than predicted by GR. However, subsequent star catalogs have been generated, including the FK5. There is only a simple rotation matrix between the FK4 and FK5 star catalogues due to Earth's general precession and the difference in epochs, but no additional algorithms exist to correct for any warped sectors within the FK4 catalog to translate into the FK5. No warped sectors within the FK4 catalog have been reported. Therefore, the forced modifications of Icarus observations to agree with GR due to a potentially warped FK4 catalog are rejected. Shapiro's result using all observations for the relativistic perihelion 
shift of the orbit of Icarus is $\lambda=0.75 \pm 0.08$ [11]. This is a significant deviation of observations with GR. Shapiro documented in detail that this study used the original Universal Time (UT) that was smoothed over the months of observations, and this test neither used any ephemeris time (ET), which was also called coordinate time (CT), nor any atomic timescale, which was then designated as A1. He used USNO bulletins that gave $\Delta \mathrm{T}=\mathrm{ET}-\mathrm{UT}$ to convert ET (or CT) to UT.

A brief background of the various timescales is reviewed in this paper. Greenwich Mean Time (GMT) began when the Greenwich observatory was established in 1676 and was corrected for Earth's elliptical orbit (requiring the equation of time in published tables). Pendulum clocks were synchronized to GMT, and it was assumed that Earth's rotation was uniform, since pendulum clocks of that period were not as precise. The international conference in 1884 recognized GMT as the international timescale and renamed it Universal Time (UT) [15]. Chandler discovered that Earth's rotation axis slightly wobbled [16], which made UT vary due to the latitude of the observatory. The modification of UT due to the Chandler wobble produces the mean UT timescale denoted as UT1. In this paper, UT and UT1 are considered synonymous. Simon Newcomb compiled the mean longitudes of the Sun over 142 years and fitted a polynomial through the data to obtain a smooth timescale used for internal calculations of the orbits of the planets [18]. Clemence proposed that this formula could be used in reverse for a timescale, later called ephemeris time (ET) [19]. This solar ET is a smooth version of UT, because the time tags are exclusively UT (alias GMT), which this formula existed 60 years prior to the invention of atomic clocks. In the early $20^{\text {th }}$ century, the best theories were from S. Newcomb for the Sun and four inner planets [17] and from E. W. Brown for the Moon [41]. One problem with Brown's lunar theory was the predictions were always ahead of lunar observations, so Brown incorporated a Great Empirical Term (GET). In 1926 and again in 1939, Spencer Jones analyzed the residuals between these theories and observations and presented the formulae for the correction of the lunar mean longitudes compared to the solar mean longitudes [25]. In the same paper earlier quoted, Clemence documented how to alter Brown's lunar theory with the appropriate corrections to account for Earth's rotational deceleration (i.e. tidal friction) that Spencer Jones documented and remove the GET [19]. Clemence's recommendations were the foundation for the Improved Lunar Ephemeris (ILE), which his proposals were approved in stages by the International Astronomical Union.

Soon afterwards, cesium clocks were developed for longterm ultraprecise timekeeping. It remained for Markowitz and Hall of the US Naval Observatory (USNO) and Essen and Perry of the National Physical Laboratory (NPL) to calibrate the cesium clocks to the ILE using lunar observations from the dual rate Moon camera [27]. The calibration was conducted from 1955.0 through 1958.25 and resulted in the definition of the Système International (SI) second, which is the basic time unit of International Atomic
Time (TAI) [27]. The lunar ET second remained within 1E$10 \mathrm{~s}$ of the SI second after 30 years of this calibration, so for all practical purposes, the atomic second (SI second) and the lunar ET second from the ILE are identical [28]. Even though TAI was set to be identical with UT on 0 January 1958, TAI steadily diverged ahead of UT. The very team that so precisely calibrated the SI second to the lunar ET second reported that the UT second was larger than the lunar ET second by using the same calibration data from 1955 through $1958[30,31]$.

It was incorrectly assumed that tidal friction was the source of the problem to make the UT second longer as time elapses [20]. Tidal friction was accurately compensated in the ILE through the research of Spencer Jones [21, 23] and de Sitter [22], and transformed correctly by Clemnce [19]. Later, Deines and Williams confirmed that the ILE had an accurate compensation for tidal friction by conservation of angular momentum transfer from lunar tides into the lunar orbit [24]. The UT second was already longer during the calibration effort, so it would not be getting significantly longer after 1958 from tidal friction. The ILE itself shows from linear terms that the lunar ET second is shorter than the UT second by approximately $2.292 \mathrm{E}-8 \mathrm{~s}$. No relativity effects were included in the ILE [29]. Deines and Williams calculated that time dilation would cause the undilated ET second to be $2.468 \mathrm{E}-8 \mathrm{~s}$ shorter than the proper second (i.e. UT second) [25]. Many time anomalies can be explained by this size difference between the original UT second and the SI second (alias lunar ET second). The excess length-of-day between UT and TAI has been a mean value of $2.1 \mathrm{~ms}$ from 1958 through 1998. Simply multiply 86400 seconds (length of a day) by $2.468 \mathrm{E}-8$. This size difference will cause a divergence of $0.7787 \mathrm{~s}$ over 1 year. From January 1958 through December 1998 (41 years), there have been 32 leap seconds inserted to account for the nearly linear divergence between UT and TAI. The size difference between the UT and SI seconds will cause a divergence of $31.93 \mathrm{~s}$ in 41 years, which is within $0.2 \%$ of the inserted leap seconds. (Beginning 1999, the International Earth Rotation Service adopted different data standards and new equations for UT, but these other time anomalies still can be explained by the different second sizes; but such detailed explanations covering 8 time anomalies are outside the scope of this paper.) The atomic timescale (or lunar ET) will be ahead of the original UT by 77.87 seconds in a Julian century (exactly 36525 UT days). With Mercury's orbit of 87.96925 days [20], this would give an artificial advance of $6.433 \% /$ cy in the Mercury perihelion analyses.

Nelson derived a PNA in the GR space-time metric and equation of motion with respect to an accelerated, rotating frame of reference in the presence of a gravitational field. He applied the results to obtain a test particle's motion with many mass points as generalized by the Einstein-InfieldHoffmann (EIH) equation of motion for a noninertial observer. The complete metric combined gravitational and inertial effects as a solution to the field equations. The gravitational terms are not simply added, due to the 
gravitational-inertial coupling. The metric was fully derived up to sixth order of $\mathrm{c}$ without a simple rotation of axes, which is often used in other PNAs. This metric fully applies to an observer on Earth's surface. There is no restriction, rotational or translational, on the motion of the observer's coordinates [35]. Unfortunately, Nelson did not derive the time dilation effect in his paper, but Deines and Williams derived it as the compensation that should have been included in the ILE [25]. For the convenience of the reader, that derivation is included in this paper. It is straightforward to calculate what the time shift would be for Mercury as viewed by an Earthbound observer. The effect is $t-\tau=34.30 \% / c y$. Since $t$ is coordinate time with the shorter undilated time unit, the timescale $t$ will run faster than proper time $\tau$. It is shown in the paper to be exactly $5 / 6$ of the GR prediction.

Le Verrier reported in 1857 that Mercury had an unexplained perihelion shift of $38 \%$ cy that exceed all Newtonian perturbations to its orbit [43]. Simon Newcomb later confirmed that Mercury's perihelion advance was $39 \%$ cy [17]. Both of them used UT, but their calculations used an old estimate for Earth's general precession and inaccurate planetary masses for the perturbations. Morrison and Ward [40, p. 201] listed that the planetary mass changes and general precession in 1967 would result in a net change of $-.22 \% / c y$ and $+1.81 \% / c y$, respectively. This may result in a revision of Le Verrier's and Newcomb's results of 36"/cy and 37 " cy. Le Verrier and Newcomb's results are closer to the PNA prediction in (8) than GR, with or without these corrections.

Clemence reported that the Mercury perihelion shift was (42.56 \pm 0.94$) " / c y[33,34]$, but Misner, Thorne, and Wheeler, renown relativists, adjusted Clemence's results withthe 1973 value of Earth's general precession. Their results for Clemence were $(41.4 \pm 0.9)$ "/ $/$, which is slightly below the GR prediction of $(43.03 \pm 0.03) \% /$ cy $[39,46]$. If one adds the 6.43 "/cy due to the artificial shift from using the equivalent of a lunar ET to the result of (8), then the PNA of 34.30 "/cy $+6.43 \% /$ cy $=40.73 \% / \mathrm{cy}$, which is within the adjusted Clemence error bounds. Morrison and Ward reported their result of $(41.9 \pm 0.5) \% / c y$, which is just slightly above the 40.73 "/cy. However, their error boundary is most likely too small, because they wrote, "This does not exclude the possibility that systematic biases in the timing of the contacts may have distorted our result. We show later that there are biases in the [correction to the motion of the node] data which probably affect that result, but it is very difficult to imagine why these biases should tend to depart as $\mathrm{T}^{2}$ over the 300 -yr period. These data cannot be reconciled with a value of the tidal acceleration of the Moon....and possible changes to the values of the orbital elements of Mercury and the Earth do not alter this conclusion" [40, p. 173]. It is obvious that Morrison and Ward uncovered a timing issue within the data, but they did not quantify the effect. As the timing errors did not fit any of the standard models, the error bounds are probably too small and should be at least double or triple their published result. Still, their result of 41.9 "/cy is closer to the combined result of (8) and (9) than GR.
In summary, the perihelion shift of Icarus is found to be $\lambda$ $=0.75 \pm 0.08$ [11], which $\lambda$ is the ratio of the observed relativistic perihelion shift compared to the GR prediction (i.e. $\lambda=1$ for GR and $\lambda=0$ for Newtonian physics). Nelson's PNA that is appropriate for an observer on Earth is exactly $5 / 6$ of the GR prediction. The PNA falls within the error boundary found by Shapiro's team, but their result excludes GR. As Shapiro et al documented, "either general relativity was incorrect or something in the theoretical model or observations differed from their presumptions" [11]. Shapiro's research team strictly used the original UT timescale. In the Mercury perihelion studies of Clemence $[33,34]$ and Morrison and Ward [40], the lunar ET timescale (alias TAI or atomic time) was used to time events and to generate the orbital positions from the ephemerides. Because time dilation was not included in the ILE that was used to calibrate the atomic clocks, the lunar ET or SI second is shorter than the original UT second by $2.4676 \mathrm{E}-8 \mathrm{~s}$ [25], which will result in TAI being ahead of the original UT timescale by $77.87 \mathrm{~s}$ after a Julian century. A shorter second will cause the orbital ephemeris to create an artificial advance of the Mercury perihelion of $6.433 \% / c y$. Misner, Thorne and Wheeler revised Clemence's observed perihelion shift for Mercury to $(41.4 \pm 0.9)$ \%/cy after using an updated 1973 value for Earth's general precession [39]. Again, adding the artificial perihelion advance to the post-Newtonian result of $34.30 \% /$ cy makes the sum be $40.73 \% / \mathrm{cy}$, which places it inside the revised Mercury perihelion range that technically excludes GR. The Morrison and Ward result has a timing issue that they did not quantify, but they used a 1967 value for Earth's precession, which could have caused their result to be slightly higher than Misner, Thorne and Wheeler's final result, who used a 1973 finding for Earth's general precession. If one strictly uses the original UT timescale, the PNA prediction is closer to the observed relativistic perihelion shift of Icarus and Mercury than GR's prediction.

\section{References}

[1] Miller, A. K., Albert Einstein's Special Theory of Relativity: Emergence and Early Interpretation, Addison-Wesley Publishing Company, Inc., 1981.

[2] Einstein, A., Jahrb. Radioakt, 4, 411 (1907).

[3] Planck, M. Stizungsber. Preuss. Akad. Wiss., (1907) p. 542.

[4] Einstein, A., Ann. Phys. Leipzig, 26 (1908).

[5] Weinberg, S., Gravitation and Cosmology: Principles and Application of the General Theory of Relativity, John Wiley and Sons, New York, 1972.

[6] Einstein, A., Sitzungsber. Preuss. Akad. Wiss., (1914) p. 1030 and (1915) pp. 778, 799, 831, 844.

[7] Einstein, A., Annalen der Phys., 49, 769 (1916).

[8] Will. C. M., Theory and Experiment in Gravitational Physics, Revised Edition, Cambridge University Press (1993) ISBN 978-0-521-43973-2. 
[9] Deines, S. D., "Timing in Simultaneity, Einstein's Train Scenario, and Precise Clock Synchronization", Int. J. of App. Math. and Theor. Phy., 2, Issue 3 (2016) p. 31-40.

[10] Deines, S. D., "Noninertial Freely Falling Frames Affected by Gravitational Tidal Forces", Int. J. of App. Math. And Theoretical Phys., 3, Issue 1(2017) p. 1-6.

[11] Shapiro, S. S., Davis, J. L., Lebach, D. E., and Gregory, J. S. "Measurement of the Solar Gravitational Deflection of Radio Waves using Geodetic Very-Long-Baseline Interferometry Data, 1979-1999", Physical Review Letters, (2004) 92, No. 12 , p. $121101-1$ to 4 .

[12] Deines, S., D., "Classical Derivation for the Total Deflection of Light", Int. J. of App. Math, and Theor. Phys., 2, 3 (2016) p. 52-56.

[13] Shapiro, I. I., Smith, W. B., Ash, M. E. and Herrick S., "General Relativity and the Orbit of Icarus", Astronomical Journal, 76, No. 7 (Sep 1971) p. 588-606.

[14] Seidelmann, P. K. (ed.), Explanatory Supplement to the Astronomical Almanac, U. S. Naval Observatory, University Science Books (1992).

[15] International Meridian Conference, Proceedings of the International Conference for a Prime Meridian and a Universal Day, (October 1 through November 1, 1884) Washington, D. C., Gibson Bros. Publishing, p. 1-213.

[16] Chandler, S. C., "On the Variation of Latitude", Astron. J. (1891) 248, p. 59-61.

[17] Newcomb, S., Astronomical Papers Prepared for the Use of the American Ephemeris and Nautical Almanac, Vol. 6, Part I: Tables of the Sun, Washington, DC: U. S. Govt. Printing Office, (1895).

[18] Newcomb, S., The Elements of the Four Inner Planets and the Fundamental Constants of Astronomy, Washington, DC: U. S. Govt. Printing Office, (1898) Chapter 2.

[19] Clemence, G. M. "On the System of Astronomical Constants", Astron. J. (1948) 53(6), p. 169-179.

[20] Seidelmann, P. K. [ed.], Explanatory Supplement to the Astronomical Almanac, U. S. Naval Observatory, University Science Books (1992).

[21] Spencer Jones, H., Ann. Cape Obs. (1932) 13 (3), p. 1.

[22] De Sitter, W., Bull. Astron. Inst. Netherlands, (1927) 4 (124) p. 21.

[23] Spencer Jones, H., MNRAS (1939) 99, p. 541.

[24] Deines, S. D. and Williams, C. A., "Determination of Earth's Rotational Deceleration Independent of Timescales" Astron. J. (2016), 151 (4), article 103, p. 1-12.

[25] Deines, S. D. and Williams, C. A., "Time Dilation and the Length of the Second: Why Timescales Diverge", Astron. J. (2007) 134, p. 64-70.

[26] Improved Lunar Ephemeris 1952-1959, U. S. Government Printing Office, Washington, D. C., (1954) \{prepared jointly by Nautical Almanac Offices of the United States of American and the United Kingdom\}.
[27] Markowitz, W., Hall, R. Essen, L., \& Parry, J. V. L., Phys. Rev. Lett. (1958) 1 (3), p. 105.

[28] Markowitz, W., in IAU Symp. 128, The Earth's Rotation and Reference Frames for Geodesy and Geodynamics, eds. A. Babcock and G. Wilkins (Dordrecht: Kluwer), (1988), p. 413.

[29] McCarthy, D. D. and Seidelmann, P. K., Time-From Earth Rotation to Atomic Physics, Wiley-VCH Verlag GmBH \& Co. Weinheim (2009).

[30] Essen, L., Parry, J. V. L., Markowitz, W., and Hall, R. (1958) "Variation in the Speed of Rotation of the Earth since June 1955”, Nature, 181, 1054.

[31] Markowitz, W. (1959) "Variations in the Rotation of the Earth: Results Obtained with the Dual-Rate Moon Camera and Photographic Zenith Tubes", Astron. J., 64, 106-113.

[32] Markowitz, W. (1968) in Telescopes, Chapter 7, Kuiper, G. P. and Middlehurst, B. M. (eds.), University of Chicago Press, p. 88-114.

[33] Clemence, G. M., "The Relativity Effect in Planetary Motions", Rev. of Mod. Physics, 19, No. 4, (Oct 1947), p. 361364.

[34] Clemence, G. M., "The Motion of Mercury, 1965-1937", Astron. Papers Am. Ephemerides, 11, (1943) p. 1-221.

[35] Nelson, R. A., General Relativity and Gravitation (1990) Vol. 22, p. 431.

[36] Einstein, A., "On the Electrodynamics of Moving Bodies" Ann. Phys., 17, p. 549-560, (1905) [translated from Perrett, W., and Jeffery, G. B., in The Principle of Relativity, Metheuen, London, 1923].

[37] Ohanian, H. C., and Ruffini, R., Gravitation and Spacetime, $2^{\text {nd }}$ ed., W. W. Norton \& Co., NY, (1994).

[38] Hughston, L. P., and Tod, K. P., Introduction to General Relativity, Cambridge University Press, London (1990).

[39] Misner, C. W., Thorne, K. S., and Wheeler, J. A., Gravitation 1973) p. 1112.

[40] Morrison, L. V., and Ward, C. G., "An Analysis of the Transits of Mercury 1677-1973”, Mon. Not. R. Astr. Soc. (1975) 173, p. 183-206.

[41] Brown, E. W., Tables of the Motion of the Moon, New Haven, Yale, Univ. Press, (1919).

[42] Fricke, W., Astr. J. (1967), 72, p. 1369.

[43] Le Verrier, U., "Lettre de M. Le Verrier à M. Faye sur la théorie de Mercure et sur le mouvement du périhélie de cette planète", Comptes rendus hebdomadaires des séances de l'Académie des science, Paris (1859) 49, pp. 379-383.

[44] Aoki, S., "Note on Variability of the Time Standard due to Relativistic Effect", Astron. J. (1964) 69, p. 221-222.

[45] Clemence, G. M., and Szebehely, V., "Annual Variation of an Atomic Clock”, Astron. J. (1967) 72, p. 1324-1326.

[46] Duncomb, R. L., Astr. Pap., Washington (1958) 16, Part 1. 